\title{
The Role of Digital Modeling and Simulation in Power Engineering Education
}

\author{
Mladen Kezunovic, Fellow, IEEE, Ali Abur, Fellow, IEEE, Garng Huang, Senior Member, IEEE, \\ Anjan Bose, Fellow, IEEE, and Kevin Tomsovic, Senior Member, IEEE
}

\begin{abstract}
This paper resulted from the research aimed at introducing modeling and simulation as a major methodological approach for enhancing power engineering education. The reasons for such an approach are explained first. Different options and uses of the modeling and simulation tools are discussed next. Several implementations of the teaching examples are outlined. The paper ends with the conclusions reached based on the study.
\end{abstract}

Index Terms-Load-flow analysis, modeling, power engineering education, power system transients, protection, simulation, stability, state estimation.

\section{INTRODUCTION}

$\mathbf{P}$ OWER engineering education is receiving a lot of attention lately due to restructuring and deregulation of the utility industry. This process has resulted in a significant turnover of the work force where quite a few professionals have either changed their jobs or retired as a consequence. The traditional employment philosophy, retaining the skillful workforce in the same job position for as long as possible, has changed and new hires are sought. This has generated renewed interest in college graduates with versatile background. Consequently, the approaches to teaching traditional power system topics need to be revisited to make sure that the new graduates are equipped with the required knowledge needed in a more competitive industry. Recognizing the need, the National Science Foundation initiated in 1997 a solicitation for research projects aimed at investigating new approaches to teaching power engineering courses. As a result, several projects were funded pursuing different ideas. This paper summarizes findings of one such project that has proposed the use of modeling and simulation as the preferred teaching approach.

The use of modeling and simulation in power engineering education is not a new concept. A number of excellent educational papers on this subject have been published. As an example, they range fromemphasizing the design of full-blown operatortraining simulators [1]-[5], to powerful graphical interfaces [6]-[11], and dedicated solutions for study of specific topics [12]-[16]. In most of the papers an emphasis was placed on developing either customized packages or adopting some conventional solution. The

\footnotetext{
Manuscript received July 24, 2003. This work was supported in part by the National Science Foundation under Grant ECS-96-19294 and in part by Electric Power Research Institute under Contract WO 8618-02.

M. Kezunovic, A. Abur, and G. Huang are with the Department of Electrical Engineering, Texas A\&M University, College Station, TX 77843-3128 (e-mail: kezunov@ee.tamu.edu; abur@ee.tamu.edu; huang@ee.tamu.edu).

A. Bose and K. Tomsovic are with the Department of Electrical Engineering, Washington State University, Pullman, WA 99164-2752 (e-mail: bose@eecs.wsu.edu; tomsovic@eecs.wsu.edu).

Digital Object Identifier 10.1109/TPWRS.2003.821002
}

study reported in this paper took a slightly different approach: it covered a variety of possible approaches indicating how a combination of different modeling and simulation concepts can be utilized to facilitate various teaching goals. As a result, three sets of teaching tools were studied and developed/adopted for teaching needs: customized toolbox, general-purpose toolbox, and commercial programs. Each of the modeling and simulation tools is either developed or selected to meet a specific teaching task and as such fits the teaching need the best.

The paper starts with a general discussion of the role of modeling and simulation in teaching. It then proceeds with adiscussion of related examples of specific issues in power engineering where modeling and simulation tools developed by the authors offerpowerful insights. Conclusions and references are given at the end.

\section{Role of Modeling AND SimUlation IN TEACHING}

Modeling and simulation were always useful in teaching any power system topic when:

a) complex physical interactions described by diverse mathematical formulations needed to be explained in a comprehensive way;

b) the feel for various sensitivities in the input/output relations had to be developed;

c) further understanding of the physical phenomena was possible due to new discoveries in the modeling area;

d) appropriate and easy-to-use computational tools were available.

However, the well-established system concepts and the way they are formulated were often too complex to explain with simple models that students would grasp quickly, and the tools were not flexible enough to look at various levels of complexity. The sensitivity studies were not easy to implement without data from real utility scenarios, which was hard to get, and the programs often could not be manipulated to calculate these sensitivities. Adequate modeling was not always available in the common literature for some of the physical phenomena/devices/controllers, which were then not adequately represented in the simulation tools. A lesser level of detail (e.g., more steady state than dynamic) had to suffice. Availability of the tools was limited and often they were either rather simple customized tools or pretty complex commercial tools, neither fitting the classroom needs.

The state of the art for modeling and simulation has advanced a long ways in the last 20 years, resulting in the ability to develop appropriate tools more rapidly, allowing customizing to the classroom needs. The most useful tools have 
been meta-languages like Matlab and easy-to-use graphics [17], [18]. Further developments in the mathematical formulations made modeling and simulation much more effective and desirable. Complexity has increased but modeling and simulation have advanced to help make the teaching more effective by finding ways of breaking down the complexity through appropriate modules and their interactions. Utility data for real-life scenarios became more available while, at the same time, programs can be easily and quickly developed that can be customized to explain some complex phenomenon using such large data sets. There was further progress in the study of models given in the open literature such that many phenomena other than power flow and stability could easily be illustrated (e.g., different dynamic modes that could then be used to test different types of controls). These more advanced technologies for development of modeling and simulation tools have lent themselves very well for improving the teaching and learning of power system phenomena. In this paper, we illustrate the above with several examples of tools specifically developed to teach certain fundamental aspects of electric power engineering.

The teaching of state estimation has traditionally been that of its theory and solution algorithms where as much of the real field engineering needed has been in the placement of metering. The illustration here shows the utilization of a custom-developed tool by which the effects of different metering placement strategy can be immediately observed. Another example is the teaching of new topics like buy-sell transactions and congestion management. A transactions-based power flow is used here to show how the new procedures for congestion management may work while illustrating such standard phenomena like loop flow. Powerful features of general-purpose simulation tool are utilized to implement this and the next example. The deployment of new models and modeling tools for representing further the details of complex, interactive physical phenomena are shown as the next example. This is done with the representation of transient, rather than phasor-based, cause-effect behavior of the power system and protective relays, which can lead to better protection during faults.

Finally, an illustration is presented of how it is possible to better teach those phenomena that require the representation of the complexity and size of real systems. Understanding of the scalability problems when going from simple to complex representations is necessary to teach both the problems of finding numerical solutions and the problems of operating a large power network. Combination of a general-purpose simulation tool and a specialized commercial program are used to illustrate these issues. The mentioned examples illustrate how the combination of versatile modeling and simulation tools enables teaching of complex issues that were not as comprehensively covered in the past due to the lack of adequate teaching tools and associated teaching methodology.

\section{IMPORTANCE OF STATE ESTIMATION FOR SYSTEM OPERATION}

State estimation function is an integral part of the energy management systems, which are responsible for the operation of the large interconnected power systems. This function processes a redundant set of measurements taken from different system locations and calculates the best estimate of the corresponding system state. As a result of deregulation, there are more frequent switching actions for reconfiguring the transmission system and the system may experience flow patterns for which is was not originally designed. As various control areas become consolidated under a single regional transmission organization (RTO), the state estimator will be required to handle larger size systems with a nonhomogenous metering configuration in different parts of the overall system. Hence, it is important for the engineer to understand the capabilities and limitations of the state estimator for a given set of network data and measurements.

\section{A. Goals in Teaching State Estimation}

The following goals are identified as important for teaching state estimation:

- introduce the concepts of observability, meter placement, bad data and topology errors, all of which affect the performance of the state estimators;

- facilitate better understanding and interpretation of the state estimation results;

- illustrate how bad data affect the estimate, and how they can be detected and identified;

- explain what observable islands are, and how they are detected and possibly merged.

\section{B. Use of Simulation Tools to Achieve the Teaching Goals}

While the above goals can be achieved via classroom teaching, the use of an interactive teaching tool, which provides instant feedback to the student's inputs, is shown to enhance the process. Here, the use of one such tool, namely the power education toolbox (PET) will be described. PET is composed of several application programs running underneath a graphical user interface [19]. It allows interactive data entry and displays results of applications graphically on the network diagram. Thus, the students can instantly observe the results of running an application for a given set of inputs, on the network diagram. The following sample exercises are given to illustrate the use of PET for teaching the above stated concepts.

Exercise 1: Design a measurement system, which will make the network observable and allow bad data identification for all measurements.

The students will be given the network data. They will first build the system diagram and then interactively place meters at various locations in the system. At any design stage, the students can activate the observability test function to visualize the unobservable branches and, hence, the observable islands. This will assist them to decide on the locations of new measurements to be added. Boundary injections of observable islands and flows along unobservable branches are possible candidates. In order to meet the second design requirement on bad data identification, the students will make sure that none of the measurements are critical. They can test this by running the state estimator function, which will indicate all critical measurements on the network diagram. 


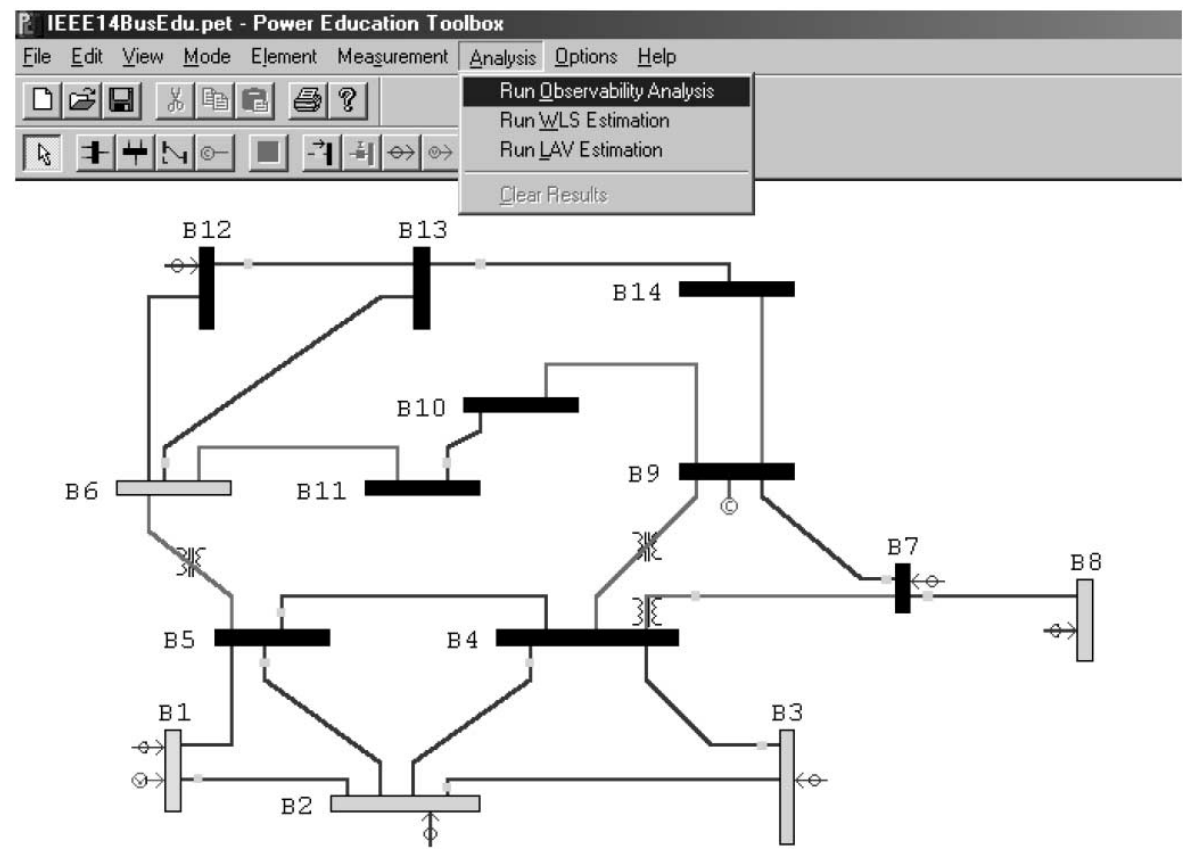

Fig. 1. PET window for exercise 1.

Fig. 1 shows a PET's window when carrying out exercise 1. Note that at this stage of the design, there are three observable islands, separated by unobservable branches, which are marked by red in the network diagram.

Exercise 2: Given a power system and its measurement configuration, edit the indicated measurement values by artificially introducing bad data and observe their effects on the estimate.

Some of these measurements will be critical and, hence, the bad data will not be detected. Some others will belong to critical n-tuples, which will allow detection but not proper identification. Finally, bad data in redundant measurements will be picked up by the identification tests. This exercise will also include a case where no measurement error will be present, but a branch will be taken out without updating the measurement set. Hence, the measurements will correspond to a different topology. This will illustrate a topology error and its impact on the state estimator. The students will apply the bad data identification and elimination procedures and learn via observation, how to interpret the results of state estimation under such conditions.

\section{Student Feedback}

PET software has been used in both undergraduate and graduate level classes in the past three years. A majority of the students indicate that having an interactive tool that is easy to learn allows them to appreciate the design aspects of engineering in addition to the analysis. Indeed, the main difference between the previous teaching style of these classes and the current one is the heavy use of engineering design examples both in homework assignments as well as classroom teaching.

\section{DifFERENCES IN PROTECTIVE RELAY BeHAVIOR WhEN PRESENTED WITH PHASORS VERSUS TRANSIENTS}

The importance of protective relaying in deregulated and restructured utility setting is even higher than before due to a more scrutinized view relay of relay misoperations in the context of financial impacts and negative consequences on customer satisfaction. The traditional teaching approach is to introduce the short-circuit methods for representing fault values and use the phasor models for studying relay design. This approach does not allow for understanding of the transient behavior of power systems and related relay reactions. Without this understanding, it is hard for a student to appreciate the differences in various relay designs that are available on the market today and to properly select and apply the designs. Today's microprocessor relays are complex controllers utilizing advanced signal processing techniques, and further insight in their operation and behavior when presented with fault transients is a critical learning experience for a future power engineer.

\section{A. Goals in Teaching Protective Relaying}

Several new teaching goals can be formulated as follows:

- understanding a difference between phasor-based solutions of a short-circuit study and waveform solution of a time-domain electromagnetic transient method;

- getting familiar with basic functions (sampling, filtering, A/D conversion) found in the front end of a digital relay;

- being able to analyze signal processing algorithms found in most common digital relaying applications;

- assessing dynamic interaction between the power system and a digital relay during faults.

\section{B. Use of Simulation Tools to Achieve the Teaching Goals}

To be able to represent digital relays and power system models in the same simulation environment so that their interaction during faults can be studied, a suitable teaching tool had to be selected. It appears that commercial software tools that are suitable for teaching are not readily available, so a new set of tools, called MERIT 2000, was developed using 


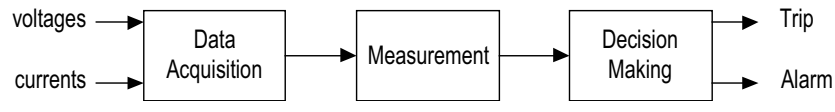

Fig. 2. Typical functional block of a digital relay.

Matlab and Simulink [20]. This approach was selected due to the popular use of Matlab and its various toolboxes in the academic community as well as due to its convenience for the study of the problem at hand since the commercial toolbox for simulating power system transient is an integral part of Matlab. New libraries for protective relay modules had to be developed and prepared for an easy use through the Simulink. As a result, several features that directly match the mentioned teaching goals are made available. This section illustrates how some of the features are utilized in the teaching exercises fulfilling the mentioned goals.

Exercise 1: Demonstrating the affect of signal processing performed by the digital relay

The following three major signal-processing blocks can represent digital relay design (see Fig. 2):

- data acquisition block that constitutes the front end of the relay and links the digital processing segment of the relay with its analog inputs;

- measuring block that estimates certain input signal parameters (magnitude, phase angle, resistance, and reactance, as well as active and reactive power);

- decision-making block that applies basic relaying principles to compare the estimated signal parameters with given settings (thresholds). It also applies certain delays and logic functions in order to issue the tripping and alarm signals.

Table I summarizes the teaching concepts that can be introduced through the use of the MERIT 2000 modules representing the three signal processing blocks.

Exercise 2: Demonstrating the dynamic interaction between a digital relay and a power system during faults

This exercise gives a unique opportunity for students to initiate faults in the power system model and to observe relay reaction to both a phasor and transient representation of the fault waveform. An example of the exercise setup and student interface provided in the MERIT 2000 tool is given in Fig. 3.

The mentioned setup allows for the following sequence of observations:

- sensitivity of the frequency content of fault waveforms to the basic fault conditions: location, fault resistance, incidence angle, fault type, loading, instrument transformer transient response, source/impedance ratio, fault infeed;

- reaction of relays (in this case, distance relay) to different fault transients, settings, and communication scheme logic

- behavior of various protective relay processing steps: signal conditioning, impedance measurement, zone logic, autoreclosing logic, out-of step logic, fault location, etc.

- dynamic reaction between power system and relays when the fault is represented with a short-circuit solution (phasors) versus electromagnetic transient solution (time-domain signal representation).
TABLE I

Teaching Signal Processing ConcePt of a Digital Relay

\begin{tabular}{|c|c|}
\hline Blocks & Signal Processing Concepts \\
\hline $\begin{array}{l}\text { Data } \\
\text { Acquisition }\end{array}$ & $\begin{array}{l}\text { Antialiasing filters, sampling theorem, } \\
\text { A/D conversion, multiplexing and signal } \\
\text { scaling, data window and sampling rate }\end{array}$ \\
\hline $\begin{array}{l}\text { Measurement } \\
\text { (Use of the men- } \\
\text { tion techniques in } \\
\text { extracting pha- } \\
\text { sors from tran- } \\
\text { sients) }\end{array}$ & $\begin{array}{l}\text { Fourier and other orthogonal transforms, } \\
\text { correlation and convolution, least square } \\
\text { Estimate, Kalman filter, differential } \\
\text { equations, recursive vs nonrecursive } \\
\text { techniques, synchronized phasor meas- } \\
\text { urements, intelligent system applications }\end{array}$ \\
\hline $\begin{array}{l}\text { Decision Making } \\
\text { (Comparison of } \\
\text { the measurement } \\
\text { to the operating } \\
\text { characteristic) }\end{array}$ & $\begin{array}{l}\text { Computation of impedance or other re- } \\
\text { laying signal, adaptive relaying, operat- } \\
\text { ing characteristic features, random time } \\
\text { delays in the relay operation driven by } \\
\text { the transient content of the input signal }\end{array}$ \\
\hline
\end{tabular}

\section{Student Feedback}

This set of modeling and simulation tools was used in teaching both the undergraduate/graduate courses as well as short courses at Texas A\&M University for the last three years. One major advantage of the configurability was the ability to select appropriate software modules that will correspond to the level of difficulty needed in a particular teaching setup. Extensive feedback from students was received in all three types of teaching environment. Invariably, the students praised the opportunity for hands-on experience in designing relays and relaying schemes that was provided through the lab exercises where the software was used. This, among other factors, led to a decision to allow for more elaborate use of the software exercises by converting the undergraduate course that was heavily relying on the use of the software to a four-credit-hour course (instead of three-credit-hour). One message for a future user (instructor) is clear: the students get very quickly frustrated if something does not work, so a day-to-day maintenance of software is needed if it is to be used efficiently.

\section{NEED FOR INCLUDING INTERDEPENDENCIES BETWEEN MARKET AND ENGINEERING ISSUES}

The power industry is implementing competitive power markets by unbundling and restructuring the vertically integrated utility into competitive entities to improve economic efficiency. However, engineering design has long been developed based on the vertically integrated system operations. The differences are drastic and there is a need to understand the fundamental issues associated with the differences. In the class, we introduce the issues by scrutinizing the power system operations and controls and comparing for differences.

\section{A. Goals in Teaching Market Issues}

Power system operation/control can be roughly divided into 13 services: Regulation, which includes load frequency control, tie-line flow control and economic dispatch; load following, which includes unit commitment; energy imbalances, which covers the power imbalances over a period of time due to load forecasting error, generator response time, and 


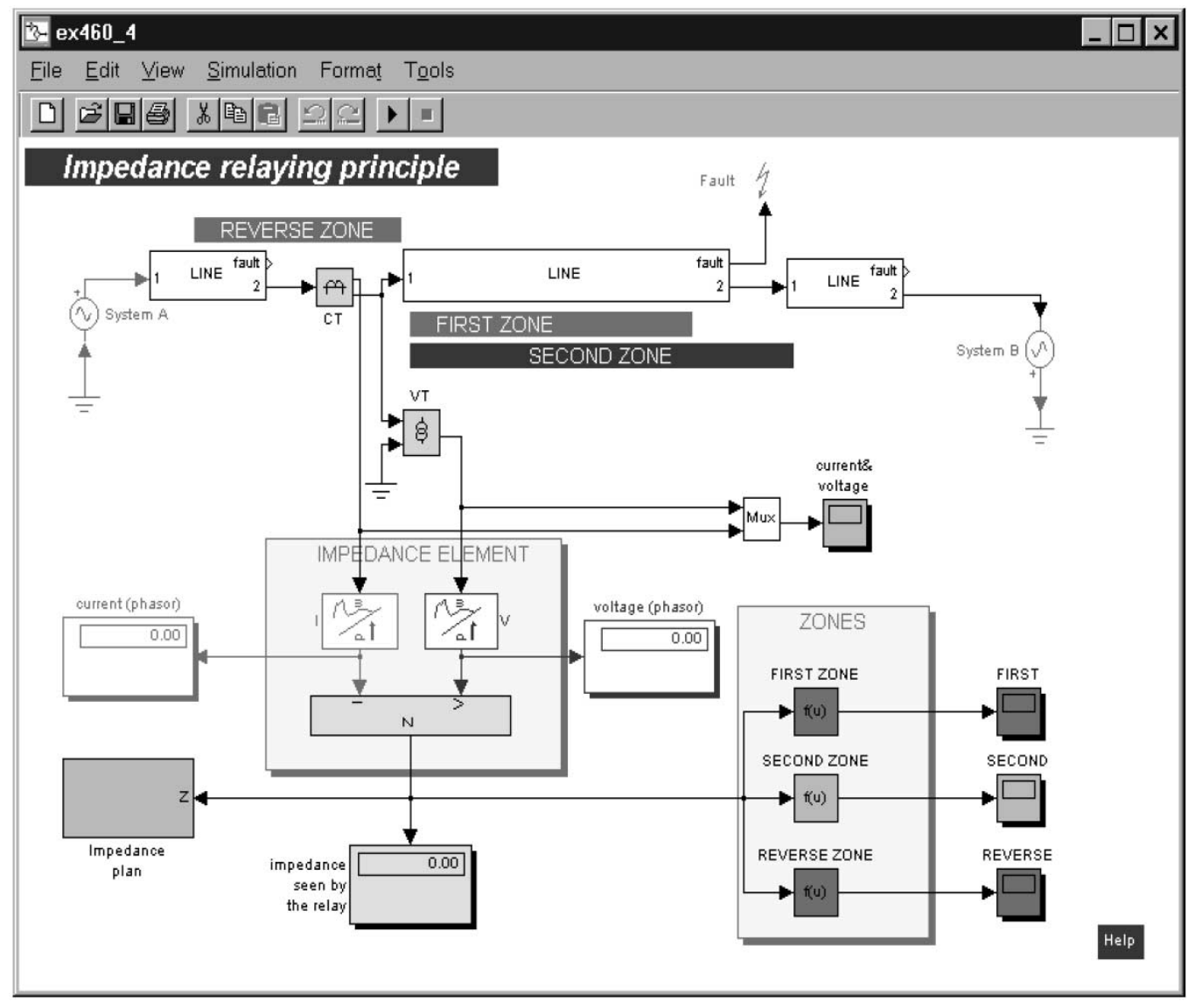

Fig. 3. MERIT 2000 interface for studies of interaction between power system and protective relay during faults.

other uncertainties; spinning operating reserve, supplemental operating reserve, and backup supply to take care of potential contingencies in a different time range; reactive power supply and voltage control; network stability services; system black start capability; power factor correction; system control to maintain generation/demand balance, transmission security, and contingencies; real-time data, telemetering, and computer interfacing, communication, etc. for dynamic scheduling; and loss replacement. In a vertically integrated system, all of the services are supplied by local utilities and coordination among neighboring utilities. The responsibility is rather clear: local utilities are responsible for whatever is deficient. However, in an unbundled deregulated market, the services are unbundled based on transactions. The complications arise and the responsibility depends on the market rules. There is a need to decompose the impacts of services based on the transactions.

Modeling and simulations become necessary to evaluate the interactions among transactions and these services to allocate responsibility and effectiveness of services for pricing purposes. The energy transactions may need to order these services for a smooth power delivery.

\section{B. Use of Simulation Tools to Achieve the Teaching Goals}

We focus here on a few illustrative issues: loss allocation and congestion responsibility evaluations. In vertically integrated utility services, loss allocation is less of an issue since power, which includes losses, is supplied by the utility generators.
Pricing is much simpler. Accordingly, a slack bus concept, which uses one generation to match all of the needed losses, is good enough an approximation since generations belong to the same local utility. However, the issue has become more complicated since now we have many generation companies supplying power to many distribution companies. The loss has to be distributed among transactions. For example, long distance transactions should incur more losses based on engineering intuition. Similarly, when congestion occurred, one needs to know which transactions are causing these flows. Accordingly, there is a need to decompose the power flow based on transactions to evaluate losses and congestion responsibilities.

Example 1: Loss allocation issues.

Advantages: ability to allocate transaction losses to transaction pairs and demonstrate different loss replacement schemes.

Example 2: Congestion issues.

Advantages: ability to decompose line flows to transaction pairs and demonstrate different transaction curtailment schemes.

In the class, the concept of bilateral transaction pairs, which consists of supplying generation and load pairs, is introduced. The market rule can require that each transaction needs to supply its own losses, then the transaction-based power flow analysis will need to evaluate the loss and, at the same time, add the loss to the transaction generation. The pricing becomes part of the contract during the bilateral transactions. The market 
rule can also use a pool market generator to supply the loss, which may end up with a similar approach as the slack bus. In addition, the power flow on each line is also decomposed based on these bilateral transactions. When a line gets congested, one can look at these decomposed flows to determine which transactions are involved and accordingly which transactions should be curtailed. Financial tools such as the flow gate right then can be easily introduced to ensure against potential transaction curtailments.

Advantages when using modeling and simulation as a tool for teaching are

- ability to evaluate different loss replacement schemes;

- ability to implement different congestion management schemes.

Software is developed on a WSCC nine-bus systems for students to evaluate different loss replacement schemes [21]. Students can develop deeper insights by comparing with conventional power flow analysis and needed changes to implement the market rules and the resultant generation and flow pattern changes. They can also evaluate different approaches of transaction curtailment to relieve a congested line. Students can use conventional power flow analysis to evaluate transaction sequentially to see the flow changes and loss changes. They will conclude that the approach becomes time consuming and unreliable when transactions are many and the incremental losses and flows will depend on how you order these transactions. The need to evaluate all losses and power decomposition in one transaction-based power flow analysis becomes clear. How to fairly allocate transaction losses without the complications of transaction ordering issues is discussed and demonstrated in the class. Modeling issues and simulation approaches then become the focus of the class.

\section{Student Feedback}

The software was used in both undergraduate and graduate level classes in the past three years. Students indicated that having an interactive tool that is easy to learn allows them to appreciate the market issues through "if and then" scenarios. The main difference between teaching courses previously and now is the heavy use of case studies both in homework assignments as well as classroom teaching to see how market rules impact the modeling, analysis, and performance.

\section{ADVANCED UNDERSTANDING OF LOAD FLOW}

Broadly speaking, the traditional power system curriculum performs poorly at addressing scaling issues with algorithms and explaining the difficulty of translating fundamental concepts into large system applications. For load flow, it is difficult, using only the mathematics and small systems, to teach fundamental modeling and algorithm issues important for large interconnected systems. There are several important modeling concerns that are often overlooked in a typical senior level course where the material must be covered in a matter of weeks. At the same time, it is these more practical application issues that quite naturally interest and, hence, motivate students to learn the material. Still, a balance must be reached between overwhelming a student with complexity and adequately covering the material.

\section{A. Goals in Teaching Load Flow}

For the study of load flow, there are essentially two interrelated computational issues: modeling and algorithmic approaches. The following fundamental concepts should be covered:

- basic bus models (i.e., slack, PV and PQ buses) and the network admittance matrix;

- general algorithmic solutions, which would normally include Gauss-Seidel, Newton-Raphson, and fast decoupled algorithms and the dc load-flow approximation.

The above can be taught in the traditional way and the students can easily implement their own algorithm in a meta-language such as Matlab on a small system. For a more insightful study, one should also address several modeling and algorithmic topics, including

- modeling issues of generator reactive power curves, phase shifters, tap changers, dc links, and remote voltage-controlled buses with the associated computational issues;

- algorithm concerns of convergence, multiple solutions, initialization, and computational efficiency including sparsity.

\section{B. Use of Simulation Tools to Achieve the Teaching Goals}

Beginning with standard material on load-flow modeling and solutions, students write Matlab code to implement algorithm solutions. These solutions are general but only tested on small textbook systems and do not include the more advanced models. Students through this exercise should have a basic understanding of the network models and the fundamental bus models. At the same time, they should have gained some understanding of numerical algorithms and the concept of convergence. This is standard material and taught much the same as done by all instructors in this area. The one difference is that students are forced to write general code and taught a few tricks of how to easily create code using a meta-language such as MATLAB.

The advanced simulation tools employed are two-pronged: educational software written by the authors in MATLAB and commercial code. The educational software is fairly sophisticated and can efficiently find solutions on systems of up to 10000 buses as well as read in several different standard file formats. Similar software written by others has been reported in the literature [22]. Source code has been organized and commented so that students can easily understand and perhaps modify the algorithm. A comparison of the analytical expressions and a sample block of code for the Newton-Raphson algorithm is given in Table II. All notations are standard. Note even without a detailed explanation of the code, the program is easily understood. The cost of this organization is that the code is neither as efficient nor as comprehensive as it might be if the full power of Matlab was employed. In contrast, the commercial code is fast and comprehensive, but suffers from requiring a steep learning curve and forces the students to rely on a black box solution. Thus, each type of software serves different educational objectives.

At this stage, students are introduced to the more sophisticated models. The models are taught but students are not expected to implement this in their code. Algorithm improvements 
TABLE II

COMPARISON OF ANALYTICAL EXPRESSIONS AND MATLAB CODE FOR NEWTON-RAPHSON LOAD FLOW

\begin{tabular}{|c|c|}
\hline Analytical Expression & Matlab Code \\
\hline \multicolumn{2}{|c|}{ For all buses, calculate the power injections based on the initial voltages } \\
\hline$S_{i}=V_{i} \sum_{j=1}^{n} Y_{i j}^{*} V_{j}^{*}$ & $\mathrm{~S}=\operatorname{diag}(\mathrm{V}) * \operatorname{conj}(\mathrm{Ybus} * \mathrm{~V})$ \\
\hline \multicolumn{2}{|c|}{ For all buses except the slack bus, calculate the real power mismatches } \\
\hline$\Delta P=P-f(V, \delta)$ & $\operatorname{deltaP}(2: \mathrm{n})=\mathrm{P}(2: \mathrm{n})-\operatorname{real}(\mathrm{S}(2: \mathrm{n})) ; \%$ Slack is bus 1 \\
\hline \multicolumn{2}{|c|}{ For all $P Q$ buses, calculate the reactive power mismatches } \\
\hline$\Delta Q=Q-g(V, \delta)$ & deltaQ(ng+1:n) $=\mathrm{Q}(\mathrm{ng}+1: \mathrm{n})-\operatorname{imag}(\mathrm{S}(\mathrm{ng}+1: \mathrm{n})) ; \% P Q$ buses start from $n g+1$ \\
\hline \multicolumn{2}{|c|}{ Main numerical iterations, continue until mismatches are small or maximum iterations exceeded } \\
\hline & while $(\max (\mathrm{abs}(\mathrm{deltaP}+\mathrm{j} *$ deltaQ $))>$ tol $) \&($ iter $<\max$ iter $)$ \\
\hline \multicolumn{2}{|c|}{ Calculate Jacobia; , Matlab Jacobian calculations not listed for brevity } \\
\hline$J=\left[\begin{array}{ll}\frac{\partial f_{i}}{\partial \delta_{j}} & \frac{\partial f_{i}}{\partial V_{j}} \\
\frac{\partial g_{i}}{\partial \delta_{j}} & \frac{\partial g_{i}}{\partial V_{j}}\end{array}\right]$ & $\mathrm{J}=\mathrm{Jacobian}$ \\
\hline \multicolumn{2}{|c|}{ Update angles at $P V$ and $P Q$ buses; update voltages at $P Q$ buses only } \\
\hline$\left[\begin{array}{l}\delta \\
V\end{array}\right]=\left[\begin{array}{l}\delta \\
V\end{array}\right]+J^{-1}\left[\begin{array}{c}\Delta P \\
\Delta Q\end{array}\right]$ & 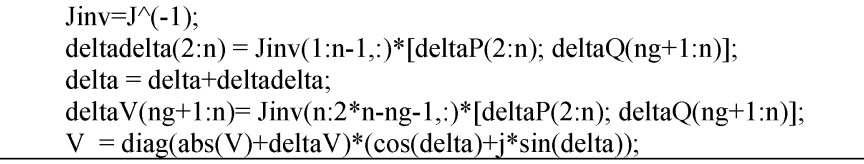 \\
\hline \multicolumn{2}{|c|}{ Calculate load flow based on new voltages and angles } \\
\hline$S_{i}=V_{i} \sum_{j=1}^{n} Y_{i j}^{*} V_{j}^{*}$ & $\mathrm{~S}=\operatorname{diag}(\mathrm{V}) * \operatorname{conj}(\mathrm{Ybus} * \mathrm{~V})$ \\
\hline \multicolumn{2}{|l|}{ Update mismatches } \\
\hline \multirow{3}{*}{$\begin{array}{l}\Delta P=P-f(V, \delta) \\
\Delta Q=Q-g(V, \delta)\end{array}$} & $\operatorname{deltaP}(2: n)=P(2: n)-\operatorname{real}(S(2: n))$ \\
\hline & $\operatorname{delta} Q(n g+1: n)=Q(n g+1: n)-i m a g(S(n g+1: n))$ \\
\hline & end; \\
\hline
\end{tabular}

are also now introduced, including sparsity and initialization issues. Students are given Matlab source code (although not necessarily all of it needs to be in source code) and the data. They are then asked to make modifications to the algorithms and/or equipment models and note the outcomes (results).

Such assignments can be thought of as a laboratory-type approach where procedures are given and students are asked to make observations. A model of the Western Electricity Coordinating Council (WECC) system of around 6000 buses has been used as it allows one to explore several issues of relevance to students at WSU. Students are asked to examine the load-flow solution during the peak and offpeak season, identifying the power consumption for several local cities, and the system transfers among regions. This teaches a feel for the Western US system. The remaining part of the assignment emphasizes both understanding the effect of certain equipment, for example, tap changing transformers, and computational issues, for example, convergence under heavy loads. The students can use the educational software to explore several other detailed algorithmic issues. For example, sparsity can be turned on and off easily. As a further example, algorithmic subtleties, such as, adaptive step sizes for convergence problems in the Newton-Raphson tech- nique, are incorporated. The approach taken in our work is to include all of these issues but spare the student the more detailed analysis and implementation issues.

The final stage in the studies is to look at analysis using commercial code. Given precise enough instructions, students can run this code without the time spent learning the program details. An alternative approach is to simply use the code in the classroom as a lecture material. Whether used as a lecture tool or part of an assignment, use of such software in a senior level course is necessarily a black box exercise. A typical demonstration with this system would show the effect of increased generation in the Northwest on flows through the California-Oregon Intertie as well as increased flow through the Rocky Mountains (i.e., loop flows). The advantage of using the commercial software over the Matlab software (which can solve systems of this size) is to overcome some of the modeling limitations. Presently, the Electric Power Research Institute (EPRI) package IPFLOW [23] has been used but other commercial programs, such as GE PSLF or PowerWorld may be more useful [10], [24]. Furthermore, the commercial code provides the students a more convincing demonstration of the system issues and at least a sense of the use of more exacting software. 


\section{Student Feedback}

Use of these methods was assessed both formally with student evaluations and informally from discussions with students. Since these methods were introduced for all students within a course, there is no good control group for statistical assessment. Students rated the material positively and course evaluations were higher after these tools were introduced but, of course, there are many factors influencing student evaluations (including the reworking of the material to accommodate the modeling). Initial student reaction was negative to the commercial software, as it required too steep of a learning curve. Subsequently, the instructor either ran the studies for the students in a classroom setting or provided "cookbook"-type directions to the students. In addition, students had difficulty ignoring the more advanced modeling components of the commercial tools that were not relevant to their studies. Students were generally more comfortable with the in-house software as they were at least generally familiar with all aspects of the modeling. Still, the commercial software elicited far more discussion and involvement of the students, particularly where students were able to relate to a specific example. For example, one of the assignments required noting the different seasonal load-flows solutions at substations within the local community. Generally, student's questions seemed to indicate a greater understanding of the application of load flow after using the commercial tools. Another final observed difficulty was the challenge in assessing student performance and understanding of the material. Simulation tools often allow the less motivated student to achieve the objectives of an assignment with minimal effort—only the more interested students truly satisfied the instructor objectives.

\section{CONCLUSIONS}

The paper discussion leads to the following conclusions.

- future students need to be exposed to "nontraditional" topics such as meter placement in state estimation, transient protective relay behavior, transaction-based congestion and loss assessment, scaling, and modeling in load-flow studies;

- modeling and simulation tools needed to teach the mentioned topics are not readily available in a single package; hence, the use of a combination of custom designed, general-purpose, and dedicated commercial modeling and simulation solutions is needed;

- using the mentioned tools, the students can grasp some fundamental teaching issues uniquely represented by modeling and simulation: competing algorithms, sensitivity study, initialization, scaling and convergence, interaction of devices and systems, modeling assumptions, observability and robustness, computational efficiency, etc.

\section{REFERENCES}

[1] Y. Tamura, E. Dan, I. Horie, Y. Nakkanishi, and S. Yokokawa, "Development of power system simulator for research and education," IEEE Trans. Power Syst., vol. 5, pp. 492-498, May 1990.

[2] B. Valiquetta, R. P. Malhamé, Y. Gervais, and D. Mukhedkar, "Microcomputer based power network control center simulator for education," IEEE Trans. Power Syst., vol. 5, pp. 474-481, May 1990.
[3] C. Y. Teo and H. B. Gooi, "A microcomputer based integrated generation and transmission system simulator," IEEE Trans. Power Syst., vol. 10, pp. 44-50, Feb. 1995.

[4] M. Foley, Y. Chen, and A. Bose, "A real time power system simulation laboratory environment," IEEE Trans. Power Syst., vol. 5, pp. 1400-1406, Nov. 1990.

[5] E. K. Tam, F. Badra, R. J. Marceau, M. A. Marin, and A. S. Malowany, "A web-based virtual environment for operator training," IEEE Trans. Power Syst., vol. 14, pp. 802-808, Aug. 1999.

[6] J. A. Huang and F. D. Galiana, "An integrated personal computer graphics environment for power system education, analysis and design," IEEE Trans. Power Syst., vol. 6, pp. 1279-1285, Aug. 1991.

[7] A. M. Gole, O. B. Nayak, T. S. Sidhu, and M. S. Sachdev, "A graphical electromagnetic simulation laboratory for power systems engineering programs," IEEE Trans. Power Syst., vol. 11, pp. 599-606, May 1996.

[8] J. Yang and M. D. Anderson, "PowerGraf: an educational software package for power systems analysis and design," IEEE Trans. Power Syst., vol. 13, pp. 1205-1210, Nov. 1998.

[9] J.-R. Shin, W.-H. Lee, and D.-H. Im, "A windows-based interactive and graphic package for the education and training of power system analysis and operation," IEEE Trans. Power Syst., vol. 14, pp. 1193-1199, Nov. 1999.

[10] Y.-Y. Hsu, C.-C. Yang, and C.-C. Su, "A personal computer based interactive software for power system operation education," IEEE Trans. Power Syst., vol. 7, pp. 1591-1597, Nov. 1992.

[11] R. Klump, D. Scholey, and T. Overbye, "An advanced visualization platform for real-time power system operations," in Proc. 14th Power Syst. Comput. Conf., Seville, Spain, June 2002.

[12] J. H. Chow and K. W. Cheung, "A toolbox for power system dynamics and control engineering education and research," IEEE Trans. Power Syst., vol. 7, pp. 1559-1564, Nov. 1992.

[13] L. Goel, C. N. Tao, and Z. B. Osman, "Software modeling of electric sub-transmission systems for educational purposes," IEEE Trans. Power Syst., vol. 11, pp. 1139-1145, Aug. 1996.

[14] J. De La Ree and J. A. Latorre, "PC based real-time simulation for teaching power generation concepts," IEEE Trans. Power Syst., vol. 5, pp. 326-330, Feb. 1990.

[15] C.-J. Wu and W.-N. Chang, "Developing a harmonics education facility in a power system simulator for power engineering education," IEEE Trans. Power Syst., vol. 12, pp. 22-29, Feb. 1997.

[16] J. A. Martinez, "How to adapt the EMTP for classroom instruction," IEEE Trans. Power Syst., vol. 7, pp. 351-358, Feb. 1992.

[17] Mathworks, Using MATLAB, 4th ed., Jan. 1999.

[18] Using SIMULINK, The Mathworks Inc., Nov. 2000.

[19] A. Abur, F. H. Magnago, and Y. Lu, "Educational toolbox for power system analysis," IEEE Comput. Appl. Power, Oct. 2000.

[20] M. Kezunović, "User friendly, open system software for teaching protective relaying application and design concepts," IEEE Trans. Power Syst., vol. 8, pp. 986-992, Aug. 2003.

[21] G. Huang and H. Zhang, "Transmission loss allocations and pricing via bilateral energy transactions," in Proc.. IEEE Summer Meet., 1999, paper no. 99 SM 013.

[22] J. Mahseredijian, F. Alvarardo, G. Rogers, and W. Long, "MATLAB's power for power systems," IEEE Comput. Appl. Power, pp. 13-19, Jan. 2001.

[23] IPFLOW User's Manual Ver. 5.0, EPRI, Palo Alto, CA, 1997.

[24] PSFL, Brochure 10107, GE Power.Com/Energy Consulting.

Mladen Kezunovic (S'77-M'80-SM'85-F'99) received the Ph.D. degree from the University of Kansas, Lawrence, in 1980.

Currently, he is the Eugene E. Webb Professor and Director of Electric Power and Power Electronics Institute at Texas A\&M University, College Station, where he has been since 1987. He is a Member of CIGRE-Paris.

Ali Abur (SM'90-F'03) received the Ph.D. degree from The Ohio State University, Columbus, in 1985.

Currently, he is a Professor at Texas A\&M University, College Station. His teaching and research interests are in computational methods used in power system analysis. 
Garng Huang (S'76-M'80-SM'85) received the B.S. and M.S. degrees in electrical engineering from National Chiao Tung University, Hsinchu, Taiwan, R.O.C., in 1975 and 1977, respectively. He received the Ph.D. degree in Systems Science and Mathematics from Washington University, St. Louis, MO, in 1980.

Currently, he is a Professor and Director of Graduate Studies in the Department of Electric Engineering at Texas A\&M University, College Station, TX.

Anjan Bose (M'68-SM'79-F'89) is the Dean of the College of Engineering and Architecture and Distinguished Professor of Electric Power Engineering at Washington State University, Pullman. He has over 30 years of experience in universities and the power industry.

Dr. Bose is has received the Power Engineering Educator Award and the Millennium Medal. He is a member of the National Academy of Engineering.
Kevin Tomsovic (M'81-SM'01) received the B.S. degree in electrical engineering from Michigan Tech. University, Houghton, in 1982, and the M.S. and $\mathrm{Ph} . \mathrm{D}$. degrees in electrical engineering from the University of Washington, Seattle, in 1984 and 1987, respectively.

Currently, he is a Professor of Electrical Engineering and Computer Science at Washington State University, Pullman. 